\title{
CRITICAL BEHAVIOR OF THE FLUX-LINE TENSION IN EXTREME TYPE-II SUPERCONDUCTORS
}

\author{
J. P. Rodriguez \\ Theoretical Division, Los Alamos National Laboratory, Los Alamos, NM 87545.*
}

\begin{abstract}
The entropic corrections to the flux-line energy of extreme type-II superconductors are computed using a schematic dual Villain model description of the flux quanta. We find that the temperature profile of the lower-critical field vanishes polynomially at the transition with an exponent $\nu \cong 2 / 3$ in the isotropic case, while it exhibits an inflection point for the case of weakly coupled layers in parallel magnetic field. It is argued that vestiges of these effects have already been observed in high-temperature superconductors.

PACS Indices: 74.20.De, 74.60.-w, 11.15.Ha, 75.10.Hk
\end{abstract}

* Permanent address: Dept. of Physics and Astronomy, California State University, Los Angeles, CA 90032. 
It is well known that type-II superconductors allow magnetic fields to penetrate in the form of flux lines quantized in units of the flux quantum, $\Phi_{0}=h c / 2 e$, for field values above the lower critical field given by $H_{c 1}(T)=4 \pi \varepsilon(T) / \Phi_{0}$, where $\varepsilon(T)$ denotes the flux-line free energy per unit length. ${ }^{1}$ The London approximation yields $\varepsilon_{0}(T)=\left(\Phi_{0} / 4 \pi \lambda_{L}\right)^{2} \ln \lambda_{L} / \xi$ for this free energy, where both the London penetration length, $\lambda_{L}$, and the coherence length, $\xi$, vary as $\left(T_{c 0}-T\right)^{-1 / 2}$ near the critical temperature, $T_{c 0}$, within the Ginzburg-Landau mean-field approximation. Spatial fluctuations of a flux line act to reduce this meanfield line tension, however, particularly near the transition temperature. Such entropic corrections to the lower critical field have been computed ${ }^{2,3}$ and observed ${ }^{4-6}$ in the case of high-temperature superconductors, where fluctuation effects are important due to the short coherence lengths and the large transition temperatures that characterize these materials.

In this paper, we shall re-compute the flux-line tension of extreme type-II superconductors $\left(\lambda_{L} \gg \xi\right)$ in the absence of magnetic field utilizing the so-called "frozen" limit of lattice superconductors first studied by Peskin. ${ }^{7-9}$ The principle idea here is to exploit the duality of quantized magnetic flux lines in superconductors to confining strings of electric flux in pure lattice gauge theories. Hence, only the entropic correction to the line tension due to flux-line wandering is accounted for. The energy functional for three-dimensional (3D) superconductors may be expressed as $^{7}$

$$
E=\rho_{s} \sum_{r, \mu}\left\{1-\cos \left[\Delta_{\mu} \phi(r)-A_{\mu}(r)\right]\right\}+\frac{1}{4 e_{0}^{2}} \sum_{r, \mu, \nu}\left[\Delta_{\mu} A_{\nu}(r)-\Delta_{\nu} A_{\mu}(r)\right]^{2},
$$

where $\phi(r)$ is the phase of the order-parameter on a cubic lattice with spacing $a^{\prime}>\xi$, and where the magnetic flux threading the plaquette at site $r$ perpendicular to the $\mu=$ $x, y, z$ direction reads $\Phi_{\mu}(r)=\left(\Phi_{0} / 2 \pi\right) \sum_{\nu, \gamma} \epsilon_{\mu \nu \gamma} \Delta_{\nu} A_{\gamma}$. Also, $\Delta_{\mu} \phi(r)=\phi(r+\hat{\mu})-$ $\phi(r)$ is the lattice difference operator. A "frozen" superconductor (FZS) is simply the thermodynamic state in the limit of infinite phase rigidity, $\rho_{s} \rightarrow \infty$. This means that the London penetration length is small in comparison to the effective lattice constant, $a^{\prime}$, and hence that flux lines do not interact. In particular, upon making the gauge transformation $A_{\mu}=A_{\mu}^{\prime}+\Delta_{\mu} \phi$, we immediately see that this limit implies $A_{\mu}^{\prime}=2 \pi m_{\mu}$, where $m_{\mu}(r)$ is an integer field. Therefore, the energy functional (1) reduces to $E_{\mathrm{FZ}}=\sum_{r, \mu}\left(2 e_{0}\right)^{-2}\left[2 \pi n_{\mu}(r)\right]^{2}$, where $n_{\mu}(r)$ is the integer field describing unit line segments of quantized flux traversing 
the plaquette $(r, \mu)$ and satisfying the continuity equation

$$
\left.\sum_{\mu} \Delta_{\mu} n_{\mu}\right|_{r}=0
$$

i.e., $n_{\mu}=\sum_{\nu, \gamma} \epsilon_{\mu \nu \gamma} \Delta_{\nu} m_{\gamma}$. The universality class of the transition into the normal state thus falls within that of the inverted 3D XY model. ${ }^{7-9}$ Employing this schematic description of flux lines in the absence of external magnetic field, with an effective lattice constant $a^{\prime} \gtrsim \lambda_{L}$, we shall demonstrate below that the lower critical field vanishes as $H_{c 1} \propto\left(T_{c}-T\right)^{\nu}$ near the transition in "frozen" lattice superconductors, where the exponent $\nu \cong 2 / 3$ corresponds to that of the $3 \mathrm{D} X Y$ model. Via an anisotropic version of this FZS model, ${ }^{10}$ we shall also show that the temperature profile for the parallel lower critical field in superconducting films with weakly coupled layers has an inflection point. Last, we argue that these effects are realized in high-temperature superconductors in the critical regime. ${ }^{4-6}$

Isotropic Superconductor. Let us then describe the zero-field thermodynamics of flux lines in $3 \mathrm{D}$ superconductors via the energy functional

$$
E_{\mathrm{FZ}} / k_{B} T=\beta \sum_{r, \mu} n_{\mu}^{2}(r)
$$

along with constraint $(2)$, where $n_{\mu}(r)$ is the integer field that represents a unit line segment of magnetic flux quanta threading the plaquette $(r, \mu)$. The energy scale above is set by the mean-field flux-line free energy, $\varepsilon_{0}(T)=\left(\Phi_{0} / 4 \pi \lambda_{0}\right)^{2}\left(1-T / T_{c 0}\right)$, and the effective lattice constant, $a^{\prime}$, yielding $\beta=a^{\prime} \varepsilon_{0}(T) / k_{B} T$. Here, $\lambda_{0}$ is on the order of the zero-temperature London penetration length, while $T_{c 0}$ denotes the mean-field critical temperature. We presume that the lattice constant satisfies $a^{\prime} \gtrsim \lambda_{L}$, which insures that the magnetic flux is quantized on the scale of an elementary plaquette. To compute the dimensionless fluxline tension, $\sigma$, we shall place a unit monopole/anti-monopole pair separated by a large distance $R \gg a^{\prime}$ along a principle axis of the cubic lattice. The partition function for the FZS system (3) in the presence of such an external monopole charge configuration, $p(r)=\delta_{r, 0}-\delta_{r, R \hat{x}}$, therefore reads

$$
Z[p]=\sum_{\left\{n_{\mu}(r)\right\}} \Pi_{r} \delta\left[\left.\sum_{\mu} \Delta_{\mu} n_{\mu}\right|_{r}-p(r)\right] \exp \left(-E_{\mathrm{FZ}} / k_{B} T\right) .
$$

Yet since $Z[p] / Z[0] \propto e^{-\beta \sigma R}$ coincides with the correlation function of the dual 3D $X Y$ model in the Villain form, ${ }^{7} C(R) \propto e^{-R / \bar{\xi}}$, the dimensionless flux-line free energy is then 
related to the correlation length, $\bar{\xi}$, of the latter by $\sigma=(\beta \bar{\xi})^{-1}$. (Henceforth, $R$ and $\bar{\xi}$ are given in units of $a^{\prime}$.) This implies that the line tension varies as $\sigma=\sigma_{0}\left(\beta-\beta_{c}\right)^{\nu}$ near the critical temperature, where $\nu \cong 2 / 3$ is the critical exponent corresponding to the divergent correlation length of the $3 \mathrm{D}$ Villain model. ${ }^{7}$ Here, $\beta_{c} \cong \frac{3}{2}$ is the critical temperature of the latter, ${ }^{9}$ while $\sigma_{0}$ is another non-universal constant of order unity. Since the dimensions of a typical flux loop are set by the divergent correlation length, $\bar{\xi}$, shortrange interactions among the flux lines on the scale of the finite mean-field penetration length should be irrelevant at criticality. This validates the FZS limit (3). The flux-line tension (or $Z[p] / Z[0]$ ) can also be computed at low temperature by permitting maximum transverse excursions in the line of one lattice spacing in any of the remaining $2(d-1)$ directions, where $d=3$ gives the dimension of the cubic lattice. This yields

$$
\sigma \cong 1-2(d-1) \beta^{-1} e^{-\beta}
$$

which agrees with the low-temperature dependence of the interface tension in the twodimensional (2D) Ising model for the case $d=2,{ }^{11}$ as it should.

The mean-field flux-line free energy in the "frozen" superconductor model $[(2)$ and $(3)]$ is therefore renormalized down by spatial fluctuations to $\varepsilon(T)=\varepsilon_{0}(T) \sigma(\beta) \propto\left(\beta-\beta_{c}\right)^{\nu}$ near the critical temperature, $T_{c}=\left(T_{c 0}^{-1}+\beta_{c} T_{0}^{-1}\right)^{-1}$, which is determined by the condition $\beta=\beta_{c}$. Here, the energy scale $k_{B} T_{0}=a^{\prime}\left(\Phi_{0} / 4 \pi \lambda_{0}\right)^{2}$ sets the size of the critical regime, $\delta T_{c}=T_{c 0}-T_{c} \sim T_{c 0}^{2} / T_{0}$. In Fig. 1, we plot the predicted profile of the lower critical field vs. temperature, which is determined by the flux-line tension in zero-field via $H_{c 1}=4 \pi \varepsilon / \Phi_{0}$ in the case of extreme type-II superconductors. Here, we have interpolated between the low-temperature and critical behaviors discussed previously. Last, we mention that a $\lambda$ transition is also expected in the specific heat vs. temperature. ${ }^{7,9}$

Layered Superconductor (Film). Consider now an anisotropic FZS model made up of $N$ weakly coupled square lattices separated by the same effective lattice constant $a^{\prime} \gtrsim \lambda_{0}$, with an energy functional given by

$$
E_{\mathrm{FZ}} / k_{B} T=\beta \sum_{l=1}^{N} \sum_{\vec{r}}|\vec{n}(\vec{r}, l)|^{2}+\gamma \beta \sum_{l=1}^{N-1} \sum_{\vec{r}}\left|n_{z}(\vec{r}, l)\right|^{2}
$$

along with constraint (2). Here, $\vec{n}=\left(n_{x}, n_{y}\right), \vec{r}$ and $l$ respectively denote the in-plane and layer indices, while $\gamma \gg 1$ denotes the anisotropy parameter. ${ }^{1}$ The smallest energy scale 
is set by the mean-field in-plane flux-line tension, ${ }^{1} \gamma^{-1} \varepsilon_{0}(T)$, and the lattice constant, yielding $\beta=\gamma^{-1} \varepsilon_{0}(T) a^{\prime} / k_{B} T$. The author has recently studied this model in the context of the dual $X Y$ model in the Villain form with a finite number of weakly coupled layers, ${ }^{10}$ where he finds a superfluid ordering transition at (inverted) temperature $\beta_{c} \sim \pi / 4$, followed by a layer-decoupling transition at higher (inverted) temperature $\beta_{*}=2 \pi$. Very similar results were obtained previously by Korshunov in his approximate study of the infinitelayer case. ${ }^{12,13}$ Specifically, the in-plane field may be expressed as $\vec{n}(\vec{r}, l)=\vec{n}^{\prime}(\vec{r}, l)-$ $\vec{n}_{-}(\vec{r}, l)+\vec{n}_{-}(\vec{r}, l-1)$, along with constraints $\vec{\nabla} \cdot \vec{n}^{\prime}=p$ and $\vec{\nabla} \cdot \vec{n}_{-}=n_{z}$, where $p(r)$ again represents the monopole distribution and $\vec{\nabla}=\left(\Delta_{x}, \Delta_{y}\right)$. Employing the potential representation $\vec{n}_{-}=-\vec{\nabla} \Phi$ factorizes the partition function (4) into $Z=Z_{\mathrm{CG}} \Pi_{l=1}^{N} Z_{\mathrm{DG}}^{(l)}$ in the limit $\gamma \rightarrow \infty,{ }^{10}$ where $Z_{\mathrm{DG}}^{(l)}$ is the partition function for the $2 \mathrm{D}$ discrete gaussian model ${ }^{14}$ that describes the $l^{\text {th }}$ layer isolated from its neighbors $\left(n_{z}=0\right)$, while the interlayer Coulomb gas factor reads

$$
\begin{aligned}
Z_{\mathrm{CG}}[p]=\sum_{\left\{n_{z}(\vec{r}, l)\right\}} \exp \left\{-\beta \sum_{l=1}^{N} \sum_{\vec{r}, \vec{r}^{\prime}}\left[n_{z}(\vec{r}, l-1)-n_{z}(\vec{r}, l)+2 p(\vec{r}, l)\right] G^{(2)}\left(\vec{r}-\vec{r}^{\prime}\right) \times\right. \\
\left.\quad \times\left[n_{z}\left(\vec{r}^{\prime}, l-1\right)-n_{z}\left(\vec{r}^{\prime}, l\right)\right]-\gamma \beta \sum_{l=1}^{N-1} \sum_{\vec{r}} n_{z}^{2}(\vec{r}, l)\right\},
\end{aligned}
$$

with the boundary layers set to $n_{z}(\vec{r}, 0)=0=n_{z}(\vec{r}, N)$. Here, $G^{(2)}=-\nabla^{-2}$ is the Greens function for the square lattice. Notice that flux lines passing vertically through all of the layers make no contribution to the energy functional above. ${ }^{15}$ In the limit of extreme anisotropy $\gamma \rightarrow \infty$, we thus find that $Z_{\mathrm{DG}}^{(l)}$ and $Z_{\mathrm{CG}}$ respectively exhibit an inverted Kosterlitz-Thouless (KT) transition at $\beta=\beta_{c}$ and a Coulomb gas ${ }^{16}$ transition at $\beta=\beta_{*} \sim 10 \beta_{c}$.

To compute the parallel flux-line tension, we again place unit oppositely charged monopoles separated by a large distance $R \gg a^{\prime}$ along a principle axis of a given layer $l$. Since the corresponding intra-layer correlation function of the dual Villain model factorizes into $C(R)=C_{\|}(R) C_{\perp}(R)$, where $C_{\|}(R)=Z_{\mathrm{DG}}^{(l)}[p] / Z_{\mathrm{DG}}^{(l)}[0]=\left(r_{0} / R\right)^{\eta_{\|}} \exp \left(-R / \bar{\xi}_{\|}\right)$ and $C_{\perp}(R)=Z_{\mathrm{CG}}[p] / Z_{\mathrm{CG}}[0]=\left(R / r_{0}\right)^{\eta_{\perp}}$, we obtain that the dimensionless parallel line tension is given by $\sigma=\left(\beta \bar{\xi}_{\|}\right)^{-1}$, where $\bar{\xi}_{\|}$denotes the correlation length of the $2 \mathrm{D}$ Villain model ${ }^{16}$ dual to the former discrete-gaussian model. ${ }^{10,14}$ Hence, this line tension vanishes as $\sigma=\sigma_{0} \exp \left[-A_{0} /\left(\beta-\beta_{c}\right)^{1 / 2}\right]$ just below the critical temperature, ${ }^{14} T_{c}=\left(T_{c 0}^{-1}+\beta_{c} \gamma T_{0}^{-1}\right)^{-1}$, 
where $\sigma_{0}$ and $A_{0}$ again are non-universal constants of order unity. A similar temperature dependence for the parallel lower-critical field in layered superconductors has been obtained via a fermion analogy for the Lawrence-Doniach model. ${ }^{13}$ The low-temperature behavior of the parallel line tension, on the other hand, is determined by Eq. (5) for $d=2$. Although the corresponding parallel flux-line free energy, $\varepsilon_{\|}(T)=\gamma^{-1} \varepsilon_{0}(T) \sigma(\beta)$, is renormalized down with respect to the mean-field result just like in the isotropic case, we also observe that it must have an inflection point as a function of temperature just below the transition, where it vanishes exponentially. This is displayed by the parallel lower-critical field $\left(H_{c 1}^{\|}\right)$in Fig. 2, which interpolates between the low-temperature and critical behaviors. The exponential suppression of $H_{c 1}^{\|}$near $T_{c}$ indicates that critical flux-line wandering is extremely violent in two dimensions.

Concerning the lower-critical field $H_{c 1}^{\perp}$ perpendicular to the layers, classical electrodynamics dictates that it must vanish in the present film geometry. However, we may fix the ends of a vertical flux line at the boundary layers $\left[n_{z}(\vec{r}, 0)=\delta_{\vec{r}, 0}=n_{z}(\vec{r}, N)\right]$ and compute its mean-squared displacement $\bar{u}_{l}^{2}=\sum_{\vec{r} \neq 0} r^{2}\left\langle n_{z}(\vec{r}, l) n_{z}(0, l)\right\rangle / \sum_{\vec{r} \neq 0}\left\langle n_{z}(\vec{r}, l) n_{z}(0, l)\right\rangle$ at a given layer $l$. In the limit of extreme anisotropy, $\gamma \rightarrow \infty$, it becomes sufficient to consider the double-layer case, $N=2$. Since the charge correlation function for the $2 \mathrm{D}$ Coulomb gas (7) satisfies $^{16}\left\langle n_{z}(0) n_{z}(\vec{r})\right\rangle \propto-r^{-2 \beta / \pi}$, we obtain $\bar{u}^{2}=a^{\prime 2}\left(\beta-\frac{1}{2} \beta_{*}\right) /\left(\beta-\beta_{*}\right)$. Exactly the same result is also obtained for pancake vortices in the absence of Josephson coupling between layers ${ }^{3,17}$ if the in-plane London penetration length $\lambda_{L}$ is chosen for the lattice constant $a^{\prime}$. The latter choice insures flux quantization at all temperatures. It also indicates that an elementary segment of vertical flux line, $n_{z}= \pm 1$, corresponds to a stack of pancakes. We may therefore interpret the lower transition temperature, $T_{*}=\left(T_{c 0}^{-1}+\beta_{*} \gamma T_{0}^{-1}\right)^{-1}$, determined by $\beta=\beta_{*}=2 \pi$ as an evaporation point for vertical flux lines (see Fig. 2), since $\bar{u}^{2}$ diverges for $T>T_{*}$. If $\gamma$ is now presumed to be large but finite, however, we must then require that the perpendicular magnetic field exceed the decoupling cross-over field at $T_{*}{ }^{18} B_{*}^{\perp} \sim \Phi_{0} / \gamma^{2} d^{2}$. This guarantees that the distance between neighboring stacks of pancake vortices be within the Josephson penetration length, $\gamma d$. Here $d$ measures the distance between adjacent Josephson-coupled planes in the underlying Lawrence-Doniach model for layered superconductivity, ${ }^{1}$ which is essentially an anisotropic version of Eq. (1). The former condition excludes the possibility that a well- 
defined Josephson vortex connects displaced stacks of pancake vortices in adjacent layers, an effect that is not accounted for by the present factorization of the layered FZS model (6) into parallel and perpendicular parts. Last, we note that compatibility with the present FZS model requires that the physical length scales satisfy $\gamma d \gg a^{\prime} \gtrsim \lambda_{0}$.

The above results for the FZS model (6) are in apparent contradiction with those of the more general anisotropic lattice superconductor model (1) obtained by Korshunov, ${ }^{12}$ who finds that layers decouple at a temperature tenfold greater than the superfluid KT transition. This paradox is resolved by observing $(i)$ that the lattice constant in such models is on the order of the coherence length, $\xi \ll \lambda_{L}$, and $(i i)$ that such decoupling of layers naturally coincides with the vanishing of the parallel lower-critical field here. Notice this means that in the regime $T_{*}<T<T_{c}$, a Meissner phase exists for in-plane magnetic fields while each layer is resistive, a feature which has been observed experimentally. ${ }^{6,13}$ The latter, however, is then consistent with the "evaporation" of vertical flux lines at $T_{*}$ obtained here. Last, we mention that the line tension for parallel vortices can be computed directly from the double-layer $X Y$ model, ${ }^{10}$ where one recovers the previous exponential temperature dependence shown in Fig. 2. ${ }^{19}$ Again, this demonstrates the consistency of the present results with those obtained from model (1) in the extreme type-II limit, $\lambda_{L} \rightarrow \infty$, which is precisely the $X Y$-model.

High-Temperature Superconductors. Let us now apply the above theory to the oxide superconductors, which are extreme type-II $\left(\lambda_{L} / \xi \sim 100\right)$ with observable fluctuation effects. ${ }^{1-6}$ Consider first bulk $\mathrm{YBa}_{2} \mathrm{Cu}_{3} \mathrm{O}_{7-y}$, which may be classified as a 3D superconductor with strong anisotropy, ${ }^{1} \gamma \sim 10$. The scaled isotropic FZS model (3) predicts a width in temperature for critical fluctuations (see Fig. 1) on the order of $\delta T_{c} \sim \gamma T_{c 0}^{2} / T_{0}$, where again $k_{B} T_{0}=\left(\Phi_{0} / 4 \pi \lambda_{0}\right)^{2} a^{\prime}$. Assuming typical parameters $T_{c 0} \sim 100 \mathrm{~K}$ and $a^{\prime} \sim \lambda_{0} \sim 10^{3} \AA$, we obtain $\delta T_{c} \sim 0.5 \mathrm{~K}$. This estimate is consistent with recent $H_{c 1}$ measurements performed by Pastoriza et al., ${ }^{4}$ who observe fluctuation effects à la Fig. 1 to within this scale. If we suppose, however, that $a^{\prime} \sim \lambda_{L}(T)=\lambda_{0}\left(T_{c 0} / \delta T_{c}\right)^{1 / 2}$, which insures flux quantization at all temperatures, then we obtain a much more modest estimate of $\delta T_{c} \sim 2.5 \mathrm{mK}$ for the size of the critical region. Also, the exponent $\nu=2 / 3$ predicted here for the critical temperature dependence of the lower-critical field is consistent with the critical temperature dependence of the London penetration length $\lambda_{L}$ per the (neutral) 3D $X Y$ model; ${ }^{1}$ 
i.e., $H_{c 1} \propto \lambda_{L}^{-2}$, with $\lambda_{L} \propto\left(T_{c}-T\right)^{-1 / 3}$. The latter critical temperature dependence has been observed in recent penetration depth measurements on this class of high-temperature superconductors. ${ }^{20}$

The oxide superconductor $\mathrm{Bi}_{2} \mathrm{Sr}_{2} \mathrm{CaCu}_{2} \mathrm{O}_{8}$, on the other hand, may be classified as a layered superconductor with extreme anisotropy, ${ }^{1} \gamma \sim 100$. The layered FZS model (6) also predicts a fluctuation regime on the order of $\delta T_{c} \sim \gamma T_{c 0}^{2} / T_{0}$ wide in temperature. Taking the previous values for $\lambda_{0}$ and $T_{c 0}$, then the choice $a^{\prime} \sim \lambda_{0}$ results in $\delta T_{c} \sim 5 \mathrm{~K}$, which is on the order of the effect (see Figs. 1 and 2 ) observed by Brawner et al. ${ }^{5}$ and by Wan et al. ${ }^{6}$ in their measurements of the field of first penetration, $H_{p}$, along the (perpendicular) c-axis for this material. On the other hand, the choice $a^{\prime} \sim \lambda_{L}(T)=\lambda_{0}\left(T_{c 0} / \delta T_{c}\right)^{1 / 2}$ for the lattice constant with the same parameter values results in a smaller estimate $\delta T_{c} \sim 0.25 \mathrm{~K}$ for the size of the critical region. More interestingly, Wan et al. also observe an inflection point in $H_{p}$ vs. $T$ on this scale. This may be a vestige of the same prediction made here for the parallel lower-critical field if geometrical demagnetization effects are presumed to be strong. In particular, only parallel flux lines contribute to the total line free energy at temperatures $T_{*}<T<T_{c}$, since the perpendicular flux lines have by then "evaporated". This scenario requires, of course, that the perpendicular magnetic field exceed the decoupling crossover scale, ${ }^{18} B_{*}^{\perp} \sim \Phi_{0} / \lambda_{J}^{2}$, where $\lambda_{J}$ represents the Josephson penetration length. Since this scale is known to diverge exponentially as $T$ approaches the decoupling transition temperature $T_{c}$ from below, ${ }^{19}$ the former cross-over scale should be negligibly small in that regime. At the evaporation point $\left(T=T_{*}\right)$, however, we have that $\lambda_{J} \sim \gamma d$. This implies a decoupling cross-over field of approximately $1 \mathrm{kG}$ in the present case, ${ }^{1}$ where $d=15 \AA$. A direct measurement of the parallel lower-critical field in such material would clearly be very useful. Last, we mention that Wan et al. have fit their results for the 2D-3D crossover line of c-axis aligned flux lines to ${ }^{6} H_{m} \propto\left(\beta-\beta_{c}\right)^{\nu}$, with $\nu=0.68$. The latter exponent is precisely that predicted for $H_{c 1}$ vs. $T$ in the isotropic FZS model (3), which suggests that $H_{m}$ is related in some way to bulk $H_{c 1}$ along the c-axis.

Finally, we note that the Coulomb gas model (7) for vertical flux-line segments indicates that the evaporation transition at $\beta^{-1}=\beta_{*}^{-1} \sim \beta_{c}^{-1} / 10$ occurring in the limit $\gamma \rightarrow \infty$ is renormalized down to $\beta^{-1}=\epsilon_{0}^{-(N-1)} \beta_{*}^{-1}$ for $\gamma$ finite, where $\epsilon_{0}-1 \propto e^{-2 \gamma \beta_{*}}$ is the polarization at the transition point due to flux-line/anti-flux-line excitations in be- 
tween consecutive layers. Hence, flux line "evaporation" in clean layered superconductors is driven to lower temperature as the c-axis thickness increases. ${ }^{21}$ If we presume the existence of strong columnar pins, however, then such isolated flux-line/anti-flux-line excitations between layers are suppressed, resulting in an unrenormalized flux-line evaporation transition. Precisely this effect has been observed in high-temperature superconductors, where the melting-evaporation-irreversibility line moves up in temperature upon the introduction of columnar pins. ${ }^{22}$

In summary, we have elucidated the critical behavior of the flux-line tension in both isotropic and layered extreme type-II superconductors $(\xi \rightarrow 0)$ utilizing the so-called "frozen" limit of a lattice model. ${ }^{7-9}$ Notably, we predict an inflection point near the transition for the parallel lower-critical field as a function of temperature in layered superconducting films. Vestiges of this effect have already appeared in quasi-2D oxide superconductors. ${ }^{6}$ It is important to remark that fluctuations in the magnitude of the superconducting orderparameter are entirely neglected within this approach, contrary to a recent treatment of the same problem. ${ }^{23}$ This is generally a valid approximation when effects due to the normal cores are negligible, like in the present limit $\xi \rightarrow 0$, and in quasi-2D systems, where phase fluctuations drive critical properties.

The author is indebted to H. Safar, M. Maley, S. Fleshler, G. Gilmer and L. Bulaevskii for valuable discussions. This work was performed under the auspices of the U.S. Department of Energy, and was supported in part by National Science Foundation grant DMR-9322427. 


\section{References}

1. G. Blatter, M.V. Feigel'man, V.B. Geshkenbein, A.I. Larkin, and V.M. Vinokur, Rev. Mod. Phys. 66, 1125 (1994), and references therein.

2. D.R. Nelson and H.S. Seung, Phys. Rev. B 39, 9153 (1989).

3. L.N. Bulaevskii, S.V. Meshkov, and D. Feinberg, Phys. Rev. B 43, 3728 (1991); L.N. Bulaevskii, M. Ledvij, and V.G. Kogan, Phys. Rev. Lett. 68, 3773 (1992).

4. H. Safar, H. Pastoriza, F. de la Cruz, D.J. Bishop, L.F. Schneemeyer, and J.V. Waszczak, Phys. Rev. B 43, 13610 (1991); H. Pastoriza, H. Safar, E. Fernández Righi, and F. de la Cruz, Physica B 194, 2237 (1994).

5. D.A. Brawner, A. Schilling, H.R. Ott, R.J. Haug, K. Ploog, and K. von Klitzing, Phys. Rev. Lett. 71, 785 (1993).

6. Y.M. Wan, S.E. Hebboul, and J.C. Garland, Phys. Rev. Lett. 72, 3867 (1994).

7. M.E. Peskin, Ann. of Phys. 113, 122 (1978).

8. P.R. Thomas and M. Stone, Nucl. Phys. B 144, 513 (1978).

9. C. Dasgupta and B.I. Halperin, Phys. Rev. Lett. 47, 1556 (1981).

10. J.P. Rodriguez, Europhys. Lett. 31, 479 (1995).

11. L. Onsager, Phys. Rev. 65, 117 (1944); M.E. Fisher and A.E. Ferdinand, Phys. Rev. Lett. 19, 169 (1967).

12. S.E. Korshunov, Europhys. Lett. 11, 757 (1990); see also G. Carneiro, Physica C 183, 360 (1991).

13. B. Horovitz, Phys. Rev B 47, 5947 (1993); 5964 (1993); Phys. Rev. Lett. 72, 1569 (1994).

14. H. van Beijeren and I. Nolden, in Structure and Dynamics of Surfaces II, edited by W. Schommers and P. von Blanckenhagen (Springer, Heidelberg, 1987).

15. A model for vortices in layered systems with a form similar to that of Eq. (7) has been recently studied by S.W. Pierson, Phys. Rev. Lett. 73, 2496 (1994).

16. J.V. José, L.P. Kadanoff, S. Kirkpatrick and D.R. Nelson, Phys. Rev. B 16, 1217 (1977).

17. J.R. Clem, Phys. Rev. B 43, 7837 (1991); K.H. Fischer, Physica C 178, 161 (1991).

18. L.I. Glazman and A.E. Koshelev, Phys. Rev. B 43, 2835 (1991).

19. J.P. Rodriguez, Los Alamos report \# LA-UR-95-1908. 
20. S. Kamal, D.A. Bonn, N. Goldenfeld, P.J. Hirschfeld, R. Liang, and W.N. Hardy, Phys. Rev. Lett. 73, 1845 (1994).

21. Arguments based on duality with the layered $X Y$ model indicate that the evaporation transition is suppressed to low temperature for $N \gtrsim \gamma^{1 / 2}$ (see ref. 10).

22. D.R. Nelson and V.M. Vinokur, Phys. Rev. B 48, 13060 (1993), and references therein.

23. G. Blatter, B. Ivlev, and H. Nordborg, Phys. Rev. B 48, 10448 (1993). 


\section{Figure Captions}

Fig. 1. Shown is a temperature profile for $H_{c 1}$ (solid line) characteristic of isotropic extreme type-II superconductors in the critical regime (shaded area). Notably, $H_{c 1}$ deviates from the mean-field result (dashed line) in an activated manner, and it approaches the transition temperature by following a polynomial law with exponent $\nu \cong 2 / 3$ (see ref. 7).

Fig. 2. The parallel lower-critical field characteristic of extreme type-II superconducting films that are composed of weakly coupled layers is plotted as a function of temperature near the phase transition. Notice that it vanishes exponentially beyond the inflection point just below $T_{c}$. Notice also that the low-temperature transition at $T_{*}$, which marks where perpendicular flux lines "evaporate", lies well inside the mean-field regime. 


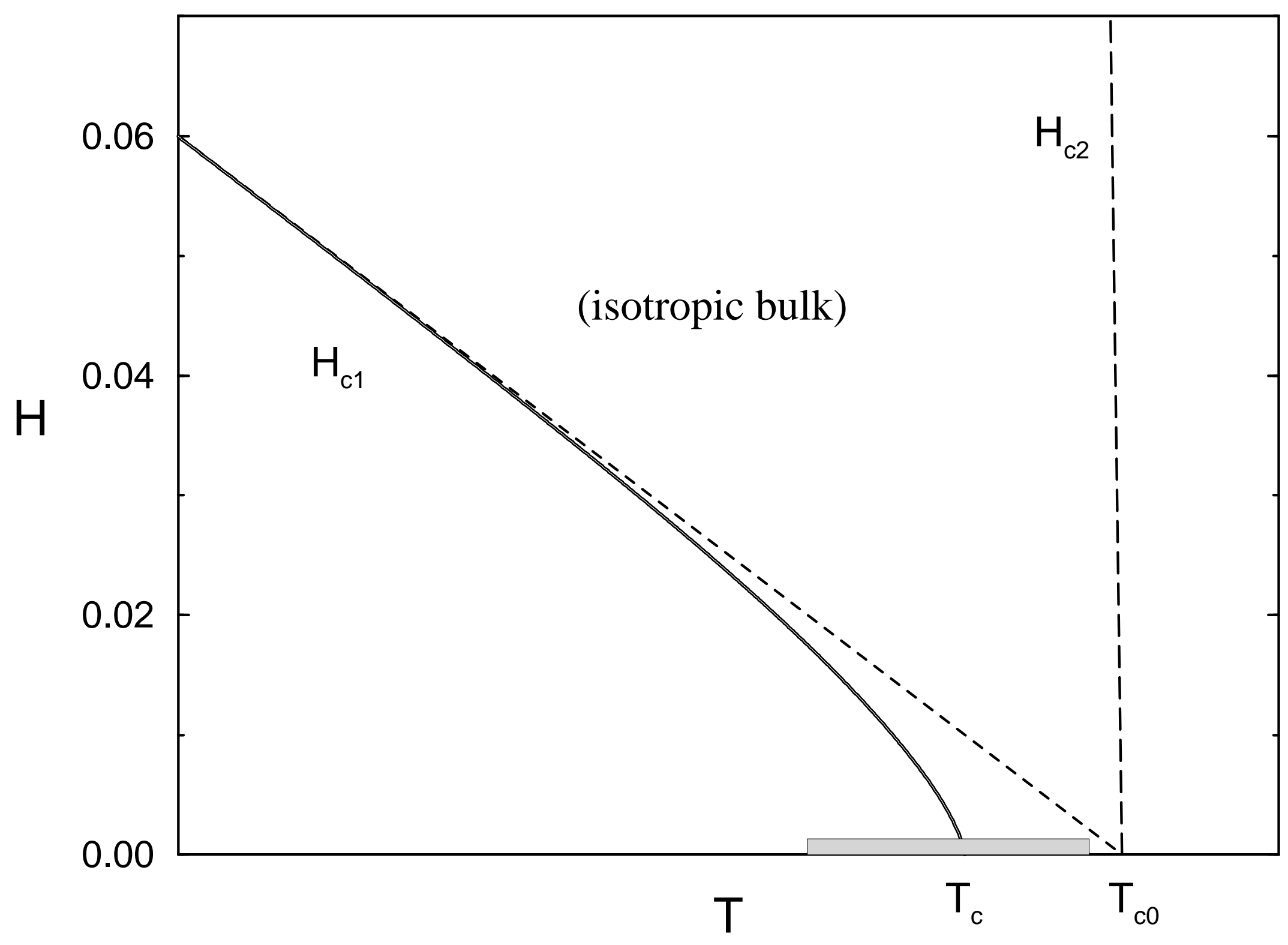




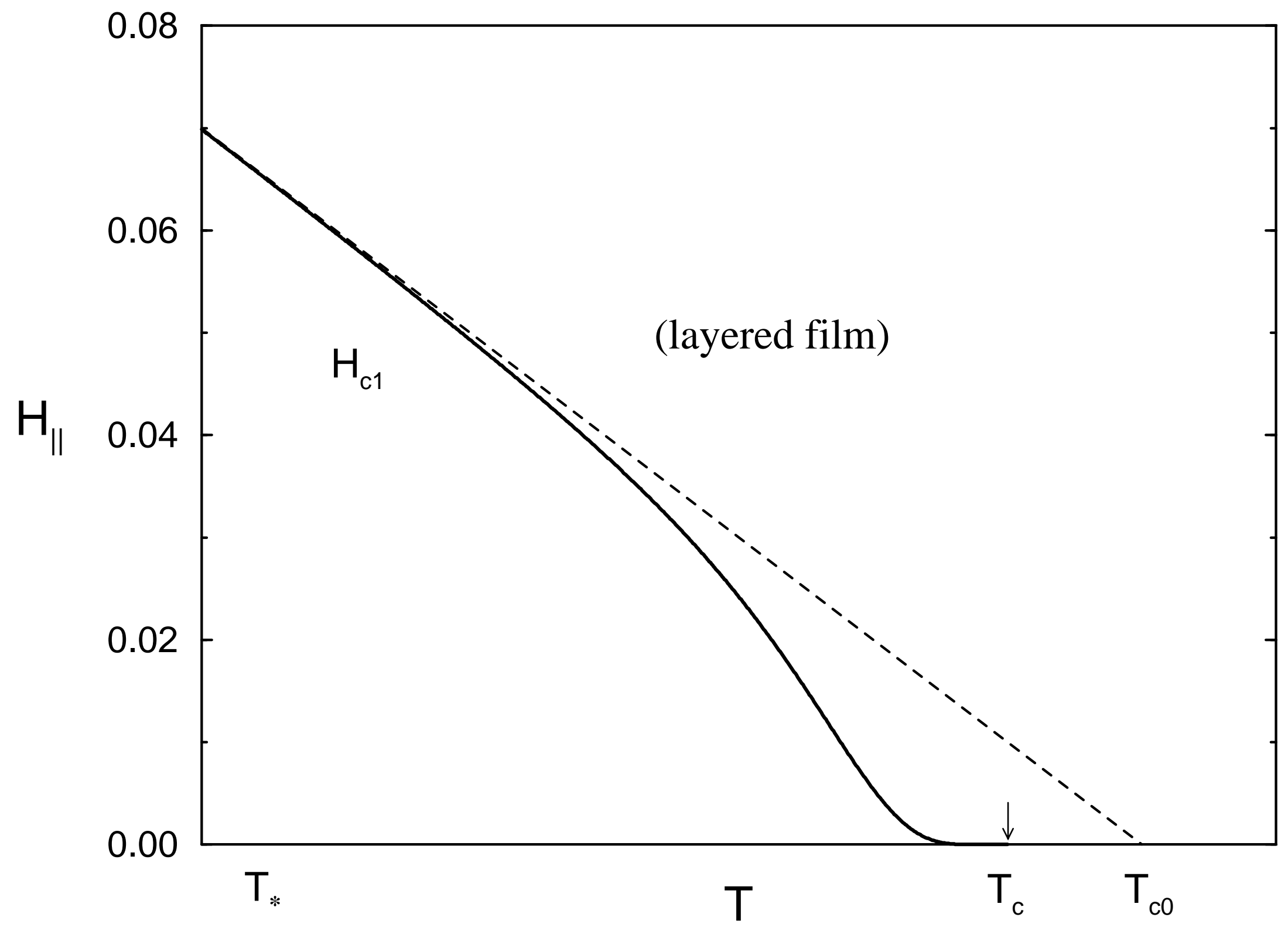

